\title{
Urate-Lowering Therapy Ameliorates Kidney Function in Type 2 Diabetes Patients With Hyperuricemia
}

\author{
Naohiko Ueno
}

\begin{abstract}
Background: Hyperuricemia often causes kidney dysfunction which increases serum urate, forming a vicious cycle in the kidney. In this study, urate-lowering therapy was demonstrated in type 2 diabetic patients with hyperuricemia to evaluate the effect on diabetic nephropathy.

Methods: Type 2 diabetic patients with hyperuricemia $(n=34)$ were treated by urate-lowering drugs. Serum urate levels, estimated glomerular filtration rate (eGFR), blood pressure, $\mathrm{HbA1c}$, and urinary albumin-to-creatinine ratio (UACR) were measured for 52 weeks. The parameters at the endpoint when serum urate decreased to below 6.0 $\mathrm{mg} / \mathrm{dL}$ and at 52 weeks were compared to the initial levels at week 0 .

Results: Serum urate level decreased to the endpoint in all patients and was maintained at under $6.0 \mathrm{mg} / \mathrm{dL}$ throughout the observation period. eGFR significantly increased at the endpoint and also at 52 weeks. Overall UACR did not change after 52 weeks; however, the treatment decreased UACR significantly in patients with no microalbuminuria. There was a negative relationship between the change of serum urate levels and the change of eGFR, and a negative relationship between the baseline UACR and the change of UACR when patients with macroalbuminuria were excluded. There were no changes in $\mathrm{HbA1c}$ levels and blood pressure before and after the treatment.
\end{abstract}

Conclusions: There were significant improvements in kidney function by lowering serum urate levels to under $6.0 \mathrm{mg} / \mathrm{dL}$ and the effect was maintained for at least 52 weeks. This treatment may be one strategy to slow the progression of nephropathy in type 2 diabetic patients with hyperuricemia.

Keywords: Hyperuricemia; Estimated glomerular filtration rate; Urinary albumin-to-creatinine ratio; Type 2 diabetes; Diabetic nephropathy

\section{Introduction}

Hyperuricemia is not just a cause of gout but is recently recog-

Manuscript submitted October 6, 2017, accepted October 23, 2017

Ueno Internal Medicine and Diabetes Clinic, Hortensia Building 5F, Motomachi-dori, Chuo-ku, Kobe, Hyogo 650-0022, Japan.

Email: uenouf3@yahoo.co.jp

doi: https://doi.org/10.14740/jocmr3219w nized as a risk factor of hypertension, cardiovascular disease, metabolic syndrome, chronic kidney disease (CKD) and diabetes [1-5]. There has been discussion as to whether uric acid is a parameter or causal factor of those pathological conditions in organs, including kidney dysfunction [6]. In fact, serum urate levels and kidney function have a close relationship. High levels of serum uric acid are risk factors for the progression of renal disease [7] and an independent predictor of new-onset CKD development [8]. On the other hand, kidney dysfunction often increases serum urate levels because the excretion of uric acid is decreased by glomerular damage. Serum urate levels increase as renal function declines [9]. Thus, hyperuricemia contributes to kidney dysfunction, and deterioration of kidney function mostly causes hyperuricemia, forming a vicious cycle. Therefore, it is necessary to break the cycle to prevent or delay the progression of kidney disease.

Steno 2 study showed that multifactorial therapy, controlling blood pressure, serum lipids and blood glucose levels is recommended in patients with diabetic nephropathy to suppress the onset and to prevent the progression of diabetic nephropathy [10]; however, the progression may be irreversible in advanced stages of the disease. Once kidney function deteriorates, drug treatment to improve kidney function has not been satisfactory.

It has been reported that hyperuricemia and diabetes have a relationship. Hyperuricemia causes insulin resistance by inhibiting insulin signaling [11], and induces oxidative damage and inhibits growth of rat pancreatic $\beta$ cells [12], suggesting a link between hyperuricemia and diabetes.

Recently, the efficacy of lowering uric acid using a single agent on kidney function has been reported [13, 14]. However, there are few reports regarding the effect of this therapy on diabetic nephropathy, and there is no strong evidence that lowering uric acid levels would prevent or slow kidney function in diabetic patients with hyperuricemia. In this study, estimated glomerular filtration rate (eGFR) and urine albumin secretion were evaluated by urate-lowering therapy in type 2 diabetic patients with hyperuricemia followed for 52 weeks.

\section{Materials and Methods}

\section{Subjects and study design}

Type 2 diabetic patients $(n=34)$ who exhibited asymptomatic hyperuricemia received one of the following urate-lowering agents: allopurinol $(\mathrm{n}=5)$, benzbromarone $(\mathrm{n}=8)$, and febux- 
ostat $(\mathrm{n}=21)$. Hyperuricemia was defined as serum uric acid $>7.0 \mathrm{mg} / \mathrm{dL}$. All patients were instructed to continue the same diet, exercise, and medication throughout the study. Patients with severe renal, liver, cardiac, neurological, hemodynamic and inflammatory diseases were excluded, and patients with pregnancy and breastfeeding were not permitted to join the study. All patients agreed by written informed consent to participate in the study. The study was conducted in accordance with the guidelines on good clinical practices and with ethical standards for human experimentation established by the Declaration of Helsinki. There is no conflict of interest related to this study.

\section{Measurements}

Serum uric acid, eGFR, blood pressure measured at clinic, and $\mathrm{HbA} 1 \mathrm{c}$ were measured monthly for 52 weeks. Urine albuminto-creatinine ratio (UACR) was determined at 0 and 52 weeks. No albuminuria, microalbuminuria and macroalbuminuria were defined as UACR $<30,30-300$, and $>300 \mathrm{mg} / \mathrm{g} \mathrm{Cr}$, respectively. All measurements were analyzed by FALCO Biosystems Ltd, Japan. The parameters at the primary endpoint, defined as serum uric acid decreasing to under $6.0 \mathrm{mg} / \mathrm{dL}$, in all patients were compared to the initial parameters at 0 and at 52 weeks. Urate-lowering drugs were used continuously throughout the study for 52 weeks, and the dosages of the drugs were adjusted (allopurinol: $200-300 \mathrm{mg} /$ day, benzbromarone: 25 - $100 \mathrm{mg} /$ day, and febuxostat: $10-40 \mathrm{mg} /$ day) to maintain serum urate levels at under $6.0 \mathrm{mg} / \mathrm{dL}$. This value of serum urate $6.0 \mathrm{mg} / \mathrm{dL}$ was defined according to the guidelines of the Japanese Society of Gout and Nucleic Acid Metabolism [15].

\section{Statistical analysis}

Results were expressed as mean \pm SE. The values of parameters before and after the treatment were analyzed using paired $t$-test. Pearson's correlation coefficients were employed to analyze the correlation of the parameters and changes. Significance was set at $\mathrm{P}<0.05$ for all analyses.

\section{Results}

At the baseline, the serum urate level was $7.8 \pm 0.1 \mathrm{mg} / \mathrm{dL}$, and eGFR was $66.0 \pm 3.1 \mathrm{~mL} / \mathrm{min} / 1.73 \mathrm{~m}^{2}$. The HbAlc level was $6.7 \pm 0.1 \%$, and systolic blood pressure and diastolic pressure were $129 \pm 2$ and $77 \pm 2 \mathrm{~mm} \mathrm{Hg}$. UACR was $83.3 \pm 30.4$ $\mathrm{mg} / \mathrm{g} \mathrm{Cr}$ (Table 1). Serum urate levels reached the endpoint in all patients. The mean duration to get to the endpoint was 9.0 \pm 2.0 weeks and the average value of serum urate at that point was $5.5 \pm 0.1 \mathrm{mg} / \mathrm{dL}$ (Fig. 1a). Due to the treatment, eGFR was significantly increased at the endpoint (from $66.9 \pm 4.6$ to $71.9 \pm 5.0 \mathrm{~mL} / \mathrm{min} / 1.73 \mathrm{~m}^{2}, \mathrm{P}<0.05$ (Fig. 1b)). Serum urate levels were maintained at under $6.0 \mathrm{mg} / \mathrm{dL}$ throughout the study for at least 52 weeks and eGFR was still significantly el-
Table 1. Baseline Characteristics of the Subjects

\begin{tabular}{|c|c|}
\hline Age & $58 \pm 2$ \\
\hline Male/female & $9 / 5$ \\
\hline BMI $\left(\mathrm{kg} / \mathrm{m}^{2}\right)$ & $26.7 \pm 0.9$ \\
\hline Serum urate (mg/dL) & $7.8 \pm 0.1$ \\
\hline eGFR $\left(\mathrm{mL} / \mathrm{min} / 1.73 \mathrm{~m}^{2}\right)$ & $66.0 \pm 3.1$ \\
\hline UACR (mg/g Cr) & $83.3 \pm 30.4$ \\
\hline HbA1c (\%) & $6.7 \pm 0.1$ \\
\hline SBP (mm Hg) & $129 \pm 2$ \\
\hline DBP (mm Hg) & $77 \pm 2$ \\
\hline Hypertension (+/-) & $26 / 8$ \\
\hline \multicolumn{2}{|l|}{ Urate-lowering agents } \\
\hline Allopurinol & 5 \\
\hline Benzbromarone & 8 \\
\hline Febuxostat & 21 \\
\hline \multicolumn{2}{|l|}{ Medication for diabetes } \\
\hline Insulin & $7(21 \%)$ \\
\hline Sulfonylurea & $10(29 \%)$ \\
\hline Glinide & $3(9 \%)$ \\
\hline GLP-1 RA & $1(3 \%)$ \\
\hline DPP-4 inhibitor & $25(74 \%)$ \\
\hline Biguanide & $17(50 \%)$ \\
\hline Thiazolidine & $7(21 \%)$ \\
\hline SGLT2 inhibitor & $6(18 \%)$ \\
\hline$\alpha \mathrm{GI}$ & $4(12 \%)$ \\
\hline \multicolumn{2}{|l|}{ Other medication } \\
\hline ARBs/ACEI & $25(74 \%)$ \\
\hline
\end{tabular}

BMI: body mass index; HbA1c: glycosylated hemoglobin A1c; eGFR: estimated glomerular filtration rate; UACR: urine albumin-to-creatinine ratio; SBP: systolic blood pressure; DBP: diastolic blood pressure; GLP1 RA: glucagon-like peptide-1 receptor agonist; DPP-4: dipeptidyl peptidase 4 inhibitor; SGLT2: sodium-glucose co-transporter 2; $a \mathrm{Gl}$ : alfa-glucosidase.

evated at 52 weeks (Table 2). When the subjects were divided into groups according to the stages of albuminuria in diabetic nephropathy, the effect of ameliorating eGFR was smaller as the stage progressed (Table 2, Fig. 1b). Overall UACR did not change in 52 weeks; however, there was a decrease in the group with no albuminuria and microalbuminuria: no albuminuria from $12.3 \pm 2.7$ to $6.6 \pm 0.9 \mathrm{mg} / \mathrm{g} \mathrm{Cr}, \mathrm{P}<0.05$, microalbuminuria from $44.1 \pm 10.8$ to $33.8 \pm 6.2 \mathrm{mg} / \mathrm{g} \mathrm{Cr}$, (Table 2, Fig. 1c). There was no effect on the macroalbuminuria group: from $402.9 \pm 121.4$ to $451.2 \pm 135.1 \mathrm{~mL} / \mathrm{min} / 1.73 \mathrm{~m}^{2}$ (Table 2, Fig. 1c).

When the effect of the drugs was evaluated, benzbromarone and febuxostat increased eGFR significantly at the endpoint (benzbromarone: from $74.7 \pm 6.9$ to $88.1 \pm 10.0 \mathrm{~mL} /$ $\mathrm{min} / 1.73 \mathrm{~m}^{2}, \mathrm{P}<0.05$; febuxostat: from $60.3 \pm 3.6$ to $64.8 \pm$ $\left.3.9 \mathrm{~mL} / \mathrm{min} / 1.73 \mathrm{~m}^{2}, \mathrm{P}<0.05\right)$ and benzbromarone still main- 

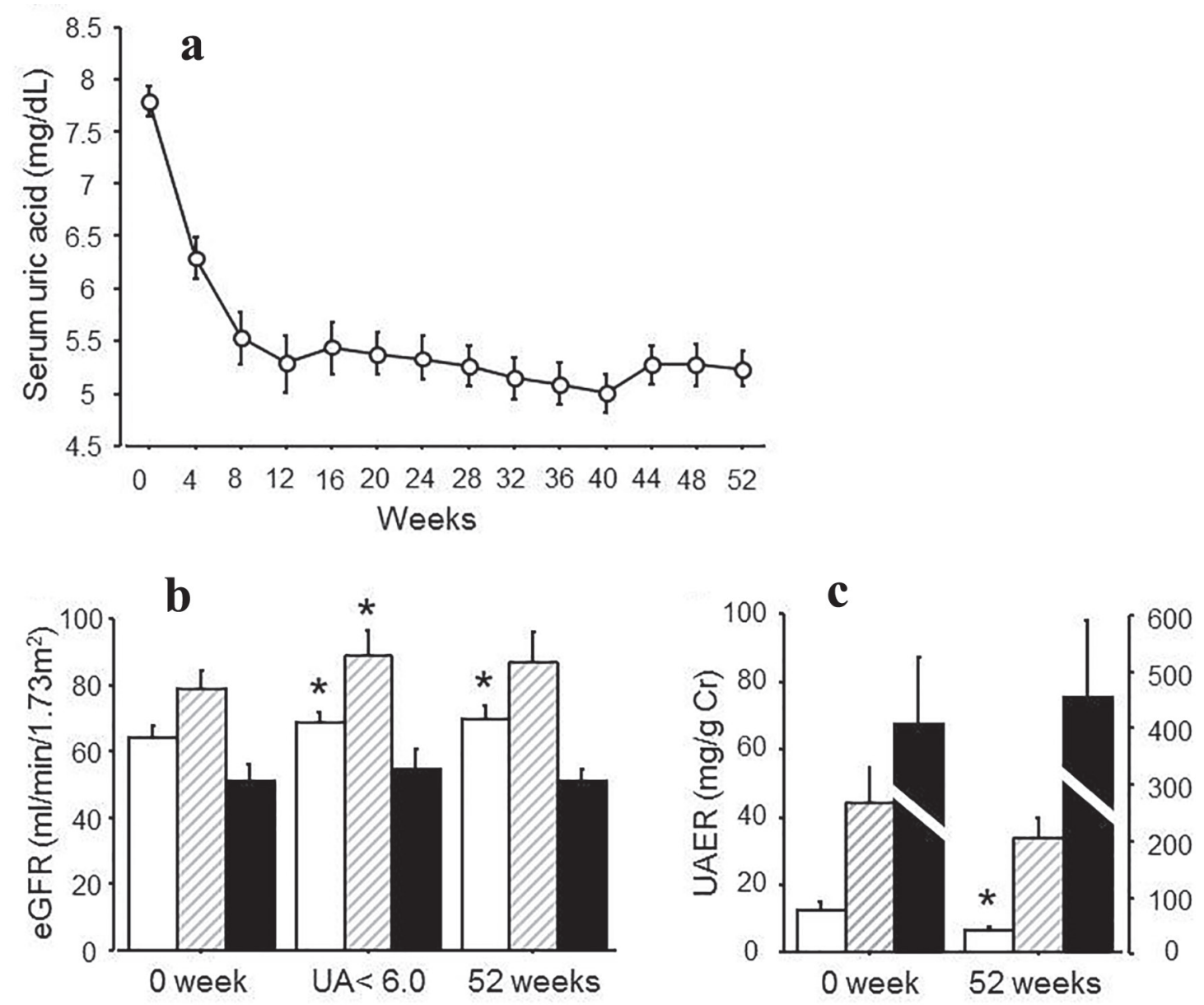

Figure 1. (a) Changes in serum urate levels after the treatment for 52 weeks. (b) eGFR at 0 week, at the point when uric acid reached under $6.0 \mathrm{mg} / \mathrm{dL}$ and at 52 weeks after the treatment. White bars: no albuminuria; shaded bars: microalbuminuria; black bars: macroalbuminuria. (c) UAER at 0 and 52 weeks after the treatment. White bars: no albuminuria; stripe bars: microalbuminuria; black bars: macroalbuminuria. ${ }^{*} \mathrm{P}<0.05$ vs. 0 week.

tained this effect for 52 weeks (Table 3). No changes in UACR were observed among those drugs (Table 3).

There was a negative relationship between the change of serum urate levels and the change of eGFR $(\mathrm{r}=-0.40, \mathrm{P}=$
0.03), and a negative relationship between the baseline UACR and the change of UACR $(\mathrm{r}=-0.76, \mathrm{P}<0.0001)$ when the patients with macroalbuminuria were excluded (Fig. 2). There were no changes in $\mathrm{HbA1c}$ levels and blood pressure before

Table 2. Changes in Serum Urate, eGFR and UACR

\begin{tabular}{|clll}
\hline & 0 week & Urate $<\mathbf{6 . 0} \mathbf{~ m g / d L}$ & $\mathbf{5 2}$ weeks \\
\hline Serum urate $(\mathrm{mg} / \mathrm{dL})$ & $7.8 \pm 0.1$ & $5.1 \pm 0.2^{*}$ & $5.2 \pm 0.2^{*}$ \\
eGFR $\left(\mathrm{mL} / \mathrm{min} / 1.73 \mathrm{~m}^{2}\right)$ & $66.0 \pm 3.1$ & $72.0 \pm 3.7^{*}$ & $71.0 \pm 3.9^{*}$ \\
No albuminuria & $64.0 \pm 3.5$ & $68.4 \pm 3.4^{*}$ & $69.5 \pm 4.0^{*}$ \\
Microalbuminuria & $78.7 \pm 5.9$ & $88.5 \pm 7.9^{*}$ & $87.1 \pm 8.5$ \\
Macroalbuminuria & $50.9 \pm 5.3$ & $54.7 \pm 5.9$ & $51.0 \pm 3.9$ \\
UACR (mg/g Cr) & $83.3 \pm 30.4$ & & $84.6 \pm 34.3$ \\
No albuminuria & $12.3 \pm 2.7$ & & $6.6 \pm 0.9^{*}$ \\
Microalbuminuria & $44.1 \pm 10.8$ & & $33.8 \pm 6.2$ \\
Macroalbuminuria & $402.9 \pm 121.4$ & & $451.2 \pm 135.1$ \\
\hline
\end{tabular}

${ }^{*} \mathrm{P}<0.05$ vs. 0 week. 
Table 3. Changes in eGFR and UACR by Urate-Lowering Agents

\begin{tabular}{|c|c|c|c|}
\hline & 0 week & Urate $<6.0 \mathrm{mg} / \mathrm{dL}$ & 52 weeks \\
\hline \multicolumn{4}{|c|}{ eGFR $\left(\mathrm{mL} / \mathrm{min} / 1.73 \mathrm{~m}^{2}\right)$} \\
\hline Allopurinol & $76.0 \pm 6.8$ & $80.0 \pm 5.5$ & $82.4 \pm 9.8$ \\
\hline Benzbromarone & $74.7 \pm 6.9$ & $88.1 \pm 10.0^{*}$ & $85.0 \pm 9.0 *$ \\
\hline Febuxostat & $60.3 \pm 3.6$ & $64.8 \pm 3.9^{*}$ & $63.1 \pm 3.9$ \\
\hline \multicolumn{4}{|l|}{ UACR (mg/g Cr) } \\
\hline Allopurinol & $22.9 \pm 9.3$ & & $13.5 \pm 6.0$ \\
\hline Benzbromarone & $9.7 \pm 1.9$ & & $15.2 \pm 6.4$ \\
\hline Febuxostat & $119.3 \pm 44.7$ & & $121.3 \pm 50.8$ \\
\hline
\end{tabular}

${ }^{*} \mathrm{P}<0.05$ vs. 0 week.

and after the treatment.

\section{Discussion}

This study demonstrates that urate-lowering therapy ameliorated kidney function in type 2 diabetic patients with hyperuricemia. The first goal of the study was to decrease serum urate levels to under $6.0 \mathrm{mg} / \mathrm{dL}$ and to evaluate kidney function. This value of serum urate was defined by Japanese guidelines for the management of hyperuricemia and gout [15] and a previous study [16]. Due to the treatment, urate levels decreased and reached the point at which uric acid levels were under $6.0 \mathrm{mg} /$ $\mathrm{dL}$ in all patients. The average duration was $9.0 \pm 2.0$ weeks after the treatment, and the average value of uric acid levels at that point was $5.5 \pm 0.1 \mathrm{mg} / \mathrm{dL}$. By lowering serum uric acid levels, significant improvement of eGFR was observed when serum uric acid levels reached the goal, and this effect was sustained for at least 52 weeks. The serum urate levels of under 6.0 $\mathrm{mg} / \mathrm{dL}$ may be an appropriate target value for controlling serum urate levels to improve kidney dysfunction in type 2 diabetes with hyperuricemia. It has been reported that the progression of kidney dysfunction is slowed by treatment with allopurinol $[13]$ or febuxostat $[14,17]$. The novel finding in this study was that not only was the progression slowed but the treatment also ameliorated kidney function, increasing eGFR. Moreover, it is noteworthy that this effect lasted for at least 52 weeks.

Diabetic nephropathy is the major cause of end-stage renal failure and a risk factor of cardiovascular disease [18]. Moreover, it remains a major cause of morbidity and mortality among diabetic patients [19]. Serum urate levels and diabetes have a close relationship and the risk of type 2 diabetes increases as serum urate levels elevate [5], and serum urate levels are associated with diabetic nephropathy [20]. It has been reported that allopurinol slows the progression of UACR compared to a control group in type 2 diabetic patients with no albuminuria [13]. In the present study, when the group was divided according to the stages of albuminuria, although no change was observed in macroalbuminuria, urinary albumin decreased significantly in the group with no albuminuria and microalbu-
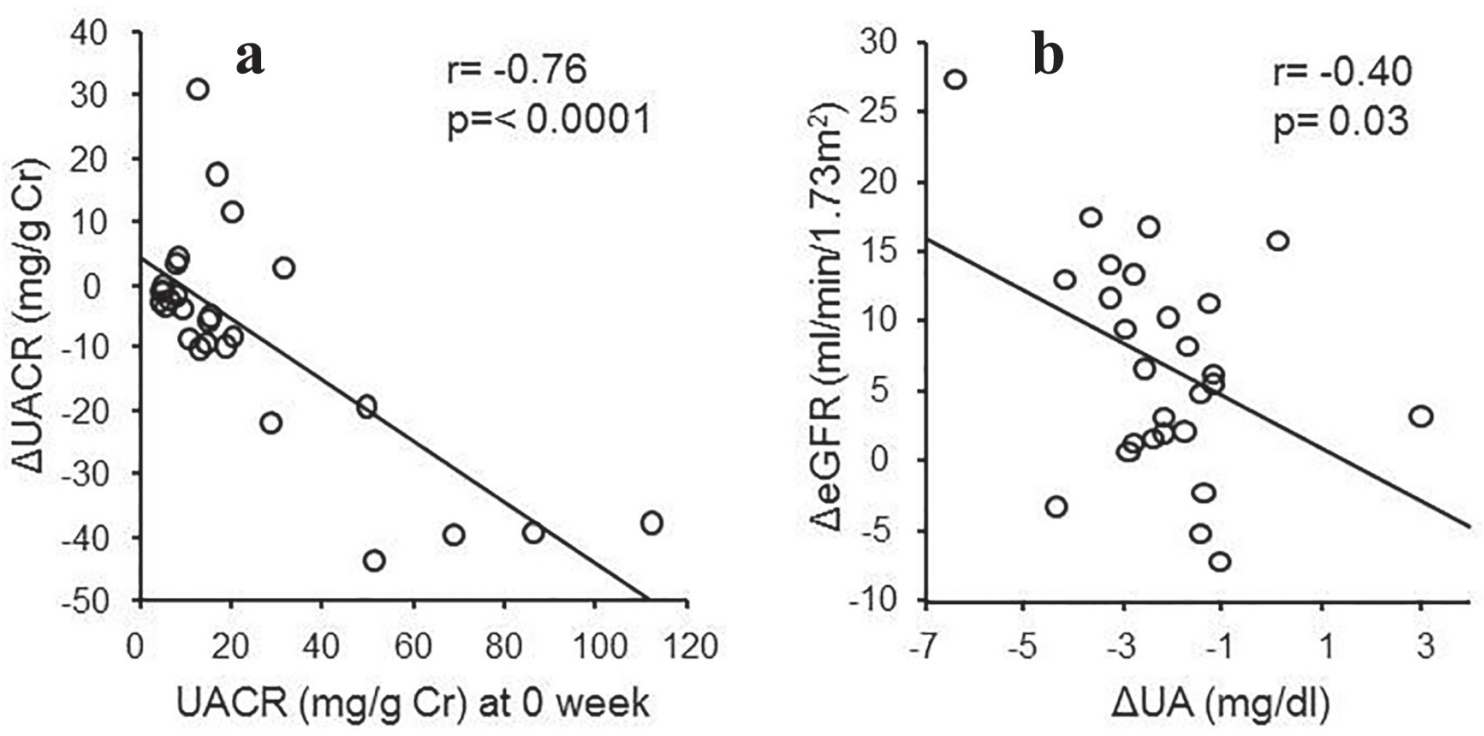

Figure 2. (a) Relationship between $\triangle \mathrm{UACR}$ and UACR at 0 week $(r=-0.76, P<0.0001)$. (b) Relationship between $\triangle$ eGFR and $\Delta \mathrm{UA}(\mathrm{r}=-0.4, \mathrm{P}=0.03)$. 
minuria. The effect on urinary albumin may be attenuated in advanced stages of diabetic nephropathy. In patients without macroalbuminuria, there was negative relationship between the baseline UACR and the change in UACR, showing that the treatment may be useful in the early stage of diabetic nephropathy for ameliorating kidney function and decreasing albuminuria. These suggest that management to lower serum urate levels should start as early as possible to prevent the progression of diabetic nephropathy.

There were improvements in eGFR and albuminuria with no changes in blood pressure and HbAlc after the treatment. Furthermore kidney function improved independently of the kind of drugs. Additionally it was shown that there was a negative relationship between the change in serum urate levels and the change in eGFR. These suggest that improvements in the kidney may be attributed to the direct effect of urate-lowering therapy.

Among the drugs used in the present study, benzbromarone, which enhances urate excretion by inhibiting the reabsorption of urate in the renal tubules [21], reduced serum urate levels the greatest. Furthermore, the greatest increase in eGFR by benzbromarone was observed. In about $90 \%$ of individuals with sustained hyperuricemia, impaired renal uric acid excretion is the dominant mechanism underlying expansion of the urate pool [22]. Accelerating the excretion to inhibit the accumulation of urate in the kidney may be more effective to ameliorate kidney dysfunction in diabetic patients with hyperuricemia.

The limitation of this study was the absence of a control group and the small number of participants. In this study, urate-lowering therapy not only slowed the progression of diabetic nephropathy but also increased eGFR, resulting in improvement of kidney function. Moreover, the treatment reduced albuminuria in the early stage of diabetic nephropathy. Interestingly, those effects were found to be dependent on the stage of diabetic nephropathy. It was less effective in the advanced stage. Controlling serum urate levels may have strong implications for reducing risks of macro- and microvascular diseases, such as diabetic complications. Early management of serum urate levels in type 2 diabetes may be one strategy to ameliorate the kidney function to some extent and may result in slowing the progression of diabetic nephropathy. Further studies should be carried out to accumulate more evidence for urate-lowering therapy in diabetic nephropathy.

\section{Conflict of Interest}

The authors declare that there is no conflict of interest.

\section{References}

1. Edwards NL. The role of hyperuricemia in vascular disorders. Curr Opin Rheumatol. 2009;21(2):132-137.

2. Ohta Y, Tsuchihashi T, Kiyohara K, Oniki H. Increased uric acid promotes decline of the renal function in hypertensive patients: a 10-year observational study. Intern Med. 2013;52(13):1467-1472.
3. Rubio-Guerra AF, Morales-Lopez H, Garro-Almendaro AK, Vargas-Ayala G, Duran-Salgado MB, Huerta-Ramirez S, Lozano-Nuevo JJ. Circulating levels of uric acid and risk for metabolic syndrome. Curr Diabetes Rev. 2017;13(1):87-90.

4. Feig DI. Uric acid: a novel mediator and marker of risk in chronic kidney disease? Curr Opin Nephrol Hypertens. 2009;18(6):526-530.

5. Bhole V, Choi JW, Kim SW, de Vera M, Choi H. Serum uric acid levels and the risk of type 2 diabetes: a prospective study. Am J Med. 2010;123(10):957-961.

6. Johnson RJ, Nakagawa T, Jalal D, Sanchez-Lozada LG, Kang DH, Ritz E. Uric acid and chronic kidney disease: which is chasing which? Nephrol Dial Transplant. 2013;28(9):2221-2228.

7. Zoppini G, Targher G, Chonchol M, Ortalda V, Abaterusso C, Pichiri I, Negri C, et al. Serum uric acid levels and incident chronic kidney disease in patients with type 2 diabetes and preserved kidney function. Diabetes Care. 2012;35(1):99-104.

8. Li L, Yang C, Zhao Y, Zeng X, Liu F, Fu P. Is hyperuricemia an independent risk factor for new-onset chronic kidney disease?: A systematic review and meta-analysis based on observational cohort studies. BMC Nephrol. 2014;15:122.

9. Ito H, Abe M, Mifune M, Oshikiri K, Antoku S, Takeuchi Y, Togane M. Hyperuricemia is independently associated with coronary heart disease and renal dysfunction in patients with type 2 diabetes mellitus. PLoS One. 2011;6(11):e27817.

10. Gaede P, Vedel P, Larsen N, Jensen GV, Parving HH, Pedersen O. Multifactorial intervention and cardiovascular disease in patients with type 2 diabetes. N Engl J Med. 2003;348(5):383-393.

11. Zhu Y, Hu Y, Huang T, Zhang Y, Li Z, Luo C, Luo Y, et al. High uric acid directly inhibits insulin signalling and induces insulin resistance. Biochem Biophys Res Commun. 2014;447(4):707-714.

12. Zhang Y, Yamamoto T, Hisatome I, Li Y, Cheng W, Sun $\mathrm{N}$, Cai B, et al. Uric acid induces oxidative stress and growth inhibition by activating adenosine monophosphate-activated protein kinase and extracellular signalregulated kinase signal pathways in pancreatic beta cells. Mol Cell Endocrinol. 2013;375(1-2):89-96.

13. Liu P, Chen Y, Wang B, Zhang F, Wang D, Wang Y. Allopurinol treatment improves renal function in patients with type 2 diabetes and asymptomatic hyperuricemia: 3 -year randomized parallel-controlled study. Clin Endocrinol (Oxf). 2015;83(4):475-482.

14. Sircar D, Chatterjee S, Waikhom R, Golay V, Raychaudhury A, Chatterjee S, Pandey R. Efficacy of febuxostat for slowing the GFR decline in patients with CKD and asymptomatic hyperuricemia: a 6-month, double-blind, randomized, placebo-controlled trial. Am J Kidney Dis. 2015;66(6):945-950.

15. Yamanaka H, Japanese Society of G, Nucleic Acid M. Japanese guideline for the management of hyperuricemia and gout: second edition. Nucleosides Nucleotides $\mathrm{Nu}-$ cleic Acids. 2011;30(12):1018-1029. 
16. Iseki $\mathrm{K}$, Ikemiya $\mathrm{Y}$, Inoue $\mathrm{T}$, Iseki $\mathrm{C}$, Kinjo $\mathrm{K}$, Takishita $\mathrm{S}$. Significance of hyperuricemia as a risk factor for developing ESRD in a screened cohort. Am J Kidney Dis. 2004;44(4):642-650.

17. Ito H, Antoku S, Abe M, Omoto T, Shinozaki M, Nishio $\mathrm{S}$, Mifune $\mathrm{M}$, et al. Comparison of the Renoprotective Effect of Febuxostat for the Treatment of Hyperuricemia between Patients with and without Type 2 Diabetes Mellitus: A Retrospective Observational Study. Intern Med. 2016;55(22):3247-3256.

18. Gross JL, de Azevedo MJ, Silveiro SP, Canani LH, Caramori ML, Zelmanovitz T. Diabetic nephropathy: diagnosis, prevention, and treatment. Diabetes Care. 2005;28(1):164-176.

19. Maahs DM, Rewers M. Editorial: Mortality and renal dis- ease in type 1 diabetes mellitus - progress made, more to be done. J Clin Endocrinol Metab. 2006;91(10):37573759.

20. Bo S, Cavallo-Perin P, Gentile L, Repetti E, Pagano G. Hypouricemia and hyperuricemia in type 2 diabetes: two different phenotypes. Eur J Clin Invest. 2001;31(4):318321.

21. Fujimori S, Ooyama K, Ooyama H, Moromizato H. Efficacy of benzbromarone in hyperuricemic patients associated with chronic kidney disease. Nucleosides Nucleotides Nucleic Acids. 2011;30(12):1035-1038.

22. Terkeltaub R, Bushinsky DA, Becker MA. Recent developments in our understanding of the renal basis of hyperuricemia and the development of novel antihyperuricemic therapeutics. Arthritis Res Ther. 2006;8(Suppl 1):S4. 